\title{
Transverse Single-Spin Asymmetries in Proton-Proton Collisions at the AFTER@LHC Experiment in a TMD Factorisation Scheme
}

\author{
M. Anselmino, ${ }^{1,2}$ U. D'Alesio, ${ }^{3,4}$ and S. Melis ${ }^{1}$ \\ ${ }^{1}$ Dipartimento di Fisica, Università di Torino, Via P. Giuria 1, 10125 Torino, Italy \\ ${ }^{2}$ INFN, Sezione di Torino, Via P. Giuria 1, 10125 Torino, Italy \\ ${ }^{3}$ Dipartimento di Fisica, Università di Cagliari, Cittadella Universitaria, 09042 Monserrato, Italy \\ ${ }^{4}$ INFN, Sezione di Cagliari, CP 170, 09042 Monserrato, Italy \\ Correspondence should be addressed to M. Anselmino; mauro.anselmino@to.infn.it
}

Received 14 April 2015; Accepted 15 June 2015

Academic Editor: Jibo He

Copyright (C) 2015 M. Anselmino et al. This is an open access article distributed under the Creative Commons Attribution License, which permits unrestricted use, distribution, and reproduction in any medium, provided the original work is properly cited. The publication of this article was funded by SCOAP $^{3}$.

\begin{abstract}
The inclusive large- $p_{T}$ production of a single pion, jet or direct photon, and Drell-Yan processes, are considered for proton-proton collisions in the kinematical range expected for the fixed-target experiment AFTER, proposed at LHC. For all these processes, predictions are given for the transverse single-spin asymmetry, $A_{N}$, computed according to a Generalised Parton Model previously discussed in the literature and based on TMD factorisation. Comparisons with the results of a collinear twist-3 approach, recently presented, are made and discussed.
\end{abstract}

\section{Introduction and Formalism}

Transverse Single-Spin Asymmetries (TSSAs) have been abundantly observed in several inclusive proton-proton experiments for a long time; when reaching large enough energies and $p_{T}$ values, their understanding from basic quark-gluon QCD interactions is a difficult and fascinating task, which has always been one of the major challenges for QCD.

In fact, large TSSAs cannot be generated by the hard elementary processes, because of helicity conservation (in the massless limit) typical of QED and QCD interactions; indeed, such asymmetries were expected to vanish at high energies. Their persisting must be related to nonperturbative properties of the nucleon structure, such as parton intrinsic and orbital motion. A true understanding of the origin of TSSAs would allow a deeper understanding of the nucleon structure.

Since the 1990s two different, despite being somewhat related, approaches have attempted to tackle the problem. One is based on the collinear QCD factorisation scheme and involves as basic quantities, which can generate single-spin dependences, higher-twist quark-gluon-quark correlations in the nucleon as well as higher-twist fragmentation correlators. The second approach is based on a physical, although unproven, generalisation of the parton model, with the inclusion, in the factorisation scheme, of transverse momentum dependent partonic distribution and fragmentation functions (TMDs), which also can generate single-spin dependences. The twist- 3 correlations are related to moments of some TMDs. We refer to [1-9], and references therein, for more detailed account of the two approaches and possible variations, with all relevant citations. Following [10], we denote by CT-3 the first approach while the second one is, as usual, denoted by GPM.

In this paper we consider TSSAs at the proposed AFTER@LHC experiment, in which high-energy protons extracted from the LHC beam would collide on a (polarised) fixed target of protons, with high luminosity. For a description of the physics potentiality of this experiment see [11] and for the latest technical details and importance for TMD studies see, for example, [12]. Due to its features the AFTER@LHC is an ideal experiment to study and understand the origin of 
SSAs and, in general, the role of QCD interactions in highenergy hadronic collisions; AFTER@LHC would be a polarised fixed-target experiment with unprecedented high luminosity.

We recall our formalism by considering the Transverse Single-Spin Asymmetry $A_{N}$, measured in $p p^{\uparrow} \rightarrow h X$ inclusive reactions and defined as

$$
A_{N}=\frac{d \sigma^{\uparrow}-d \sigma^{\downarrow}}{d \sigma^{\uparrow}+d \sigma^{\downarrow}} \quad \text { with } d \sigma^{\uparrow, \downarrow} \equiv \frac{E_{h} d \sigma^{p p^{\uparrow, \downarrow} \rightarrow h X}}{d^{3} \mathbf{p}_{h}},
$$

where $\uparrow, \downarrow$ are opposite spin orientations perpendicular to the $x-z$ scattering plane, in the $p p^{\uparrow}$ c.m. frame. We define the $\uparrow$ direction as the $+\widehat{y}$-axis and the unpolarised proton is moving along the $+\widehat{z}$-direction. In such a process the only large scale is the transverse momentum $p_{T}=\left|\left(\mathbf{p}_{h}\right)_{x}\right|$ of the final hadron.

In the GPM $A_{N}$ originates mainly from two spin and transverse momentum effects, one introduced by Sivers in the partonic distributions $[13,14]$ and one by Collins in the parton fragmentation process [15], being all the other effects strongly suppressed by azimuthal phase integrations [16]. According to the Sivers effect the number density of unpolarised quarks $q$ (or gluons) with intrinsic transverse momentum $\mathbf{k}_{\perp}$ inside a transversely polarised proton $p^{\uparrow}$, with three-momentum $\mathbf{P}$ and spin polarisation vector $\mathbf{S}$, can be written as

$$
\begin{aligned}
\widehat{f}_{q / p^{\uparrow}}\left(x, \mathbf{k}_{\perp}\right)= & f_{q / p}\left(x, k_{\perp}\right) \\
& +\frac{1}{2} \Delta^{N} f_{q / p^{\uparrow}}\left(x, k_{\perp}\right)\left(\mathbf{S} \cdot\left(\widehat{\mathbf{P}} \times \widehat{\mathbf{k}}_{\perp}\right)\right),
\end{aligned}
$$

where $x$ is the proton light-cone momentum fraction carried by the quark, $f_{q / p}\left(x, k_{\perp}\right)$ is the unpolarised TMD $\left(k_{\perp}=\left|\mathbf{k}_{\perp}\right|\right)$, and $\Delta^{N} f_{q / p^{\uparrow}}\left(x, k_{\perp}\right)$ is the Sivers function. $\widehat{\mathbf{P}}=\mathbf{P} /|\mathbf{P}|$ and $\widehat{\mathbf{k}}_{\perp}=$ $\mathbf{k}_{\perp} / k_{\perp}$ are unit vectors. Notice that the Sivers function is most often denoted as $f_{1 T}^{\perp q}\left(x, k_{\perp}\right)$ [17]; this notation is related to ours by [18]

$$
\Delta^{N} f_{q / p^{\uparrow}}\left(x, k_{\perp}\right)=-\frac{2 k_{\perp}}{m_{p}} f_{1 T}^{\perp q}\left(x, k_{\perp}\right),
$$

where $m_{p}$ is the proton mass.

Similarly, according to the Collins effect the number density of unpolarised hadrons $h$ with transverse momentum $\mathbf{p}_{\perp}$ resulting in the fragmentation of a transversely polarised quark $q^{\uparrow}$, with three-momentum $\mathbf{q}$ and spin polarisation vector $\mathbf{S}_{q}$, can be written as

$$
\begin{aligned}
\widehat{D}_{q^{\uparrow} / h}\left(z, \mathbf{p}_{\perp}\right)= & D_{h / q}\left(z, p_{\perp}\right) \\
& +\frac{1}{2} \Delta^{N} D_{q^{\uparrow} / h}\left(z, p_{\perp}\right)\left(\mathbf{S}_{q} \cdot\left(\widehat{\mathbf{q}} \times \widehat{\mathbf{p}}_{\perp}\right)\right),
\end{aligned}
$$

where $z$ is the parton light-cone momentum fraction carried by the hadron, $D_{h / q}\left(z, p_{\perp}\right)$ is the unpolarised TMD $\left(p_{\perp}=\right.$ $\left.\left|\mathbf{p}_{\perp}\right|\right)$, and $\Delta^{N} D_{q^{\uparrow} / h}\left(z, p_{\perp}\right)$ is the Collins function. $\widehat{\mathbf{q}}=\mathbf{q} /|\mathbf{q}|$ and $\widehat{\mathbf{p}}_{\perp}=\mathbf{p}_{\perp} / p_{\perp}$ are unit vectors. Notice that the Collins function is most often denoted as $H_{1}^{\perp q}\left(z, p_{\perp}\right)$ [17]; this notation is related to ours by [18]

$$
\Delta^{N} D_{h / q^{\uparrow}}\left(z, p_{\perp}\right)=\frac{2 p_{\perp}}{z M_{h}} H_{1}^{\perp q}\left(z, p_{\perp}\right),
$$

where $M_{h}$ is the hadron mass.
According to the GPM formalism $[1,2,16], A_{N}$ can then be written as

$$
A_{N}=\frac{\left[d \sigma^{\uparrow}-d \sigma^{\downarrow}\right]_{\text {Sivers }}+\left[d \sigma^{\uparrow}-d \sigma^{\downarrow}\right]_{\text {Collins }}}{d \sigma^{\uparrow}+d \sigma^{\downarrow}} .
$$

The Collins and Sivers contributions were recently studied, respectively, in [1] and [2], and are given by

$$
\begin{aligned}
& {\left[d \sigma^{\uparrow}-d \sigma^{\downarrow}\right]_{\text {Sivers }}} \\
& =\sum_{a, b, c, d} \int \frac{d x_{a} d x_{b} d z}{16 \pi^{2} x_{a} x_{b} z^{2} s} d^{2} \mathbf{k}_{\perp a} d^{2} \mathbf{k}_{\perp b} d^{3} \mathbf{p}_{\perp} \delta\left(\mathbf{p}_{\perp} \cdot \widehat{\mathbf{p}}_{c}\right) \\
& \cdot J\left(p_{\perp}\right) \delta(\widehat{s}+\widehat{t}+\widehat{u}) \Delta^{N} f_{a / p^{\uparrow}}\left(x_{a}, k_{\perp a}\right) \cos \left(\phi_{a}\right) \\
& \cdot f_{b / p}\left(x_{b}, k_{\perp b}\right) \frac{1}{2}\left[\left|\widehat{M}_{1}^{0}\right|^{2}+\left|\widehat{M}_{2}^{0}\right|^{2}+\left|\widehat{M}_{3}^{0}\right|^{2}\right]_{a b \rightarrow c d} \\
& \cdot D_{h / c}\left(z, p_{\perp}\right), \\
& {\left[d \sigma^{\uparrow}-d \sigma^{\downarrow}\right]_{\text {Collins }}} \\
& =\sum_{q_{a}, b, q_{c}, d} \int \frac{d x_{a} d x_{b} d z}{16 \pi^{2} x_{a} x_{b} z^{2} s} d^{2} \mathbf{k}_{\perp a} d^{2} \mathbf{k}_{\perp b} d^{3} \mathbf{p}_{\perp} \delta\left(\mathbf{p}_{\perp}\right. \\
& \left.\cdot \widehat{\mathbf{p}}_{c}\right) J\left(p_{\perp}\right) \delta(\widehat{s}+\widehat{t}+\widehat{u}) \Delta q_{a}\left(x_{a}, k_{\perp a}\right) \cos \left(\phi_{a}+\varphi_{1}\right. \\
& \left.-\varphi_{2}+\phi_{\pi}^{H}\right) f_{b / p}\left(x_{b}, k_{\perp b}\right)\left[\widehat{M}_{1}^{0} \widehat{M}_{2}^{0}\right]_{q_{a} b \rightarrow q_{c} d} \\
& \cdot \Delta^{N} D_{h / q_{c}^{\uparrow}}\left(z, p_{\perp}\right) .
\end{aligned}
$$

For details and full explanation of the notations in the above equations we refer to [16] (where $\mathbf{p}_{\perp}$ is denoted as $\left.\mathbf{k}_{\perp C}\right)$. It suffices to notice here that $J\left(p_{\perp}\right)$ is a kinematical factor, which at $\mathcal{O}\left(p_{\perp} / E_{h}\right)$ equals 1 . The phase factor $\cos \left(\phi_{a}\right)$ in (7) originates directly from the $\mathbf{k}_{\perp}$ dependence of the Sivers distribution $\left[\mathbf{S} \cdot\left(\widehat{\mathbf{P}} \times \widehat{\mathbf{k}}_{\perp}\right),(2)\right]$. The (suppressing) phase factor $\cos \left(\phi_{a}+\varphi_{1}-\varphi_{2}+\phi_{\pi}^{H}\right)$ in (8) originates from the $\mathbf{k}_{\perp}$ dependence of the unintegrated transversity distribution $\Delta_{T} q$, the polarized elementary interaction, and the spin- $\mathbf{p}_{\perp}$ correlation in the Collins function. The explicit expressions of $\varphi_{1}, \varphi_{2}$, and $\phi_{\pi}^{H}$ in terms of the integration variables can be found via (60)-(63) in [16] and (35)-(42) in [19].

The $\widehat{M}_{i}^{0}$ 's are the three independent hard scattering helicity amplitudes describing the lowest order QCD interactions. The sum of their moduli squared is related to the elementary unpolarised cross section $d \widehat{\sigma}^{a b \rightarrow c d}$; that is,

$$
\frac{d \widehat{\sigma}^{a b \rightarrow c d}}{d \widehat{t}}=\frac{1}{16 \pi \widehat{s}^{2}} \frac{1}{2} \sum_{i=1}^{3}\left|\widehat{M}_{i}^{0}\right|^{2}
$$

The explicit expressions of the combinations of $\widehat{M}_{i}^{0}$ 's which give the QCD dynamics in (7) and (8), can be found, for all possible elementary interactions, in [16] (see also [1] for a 

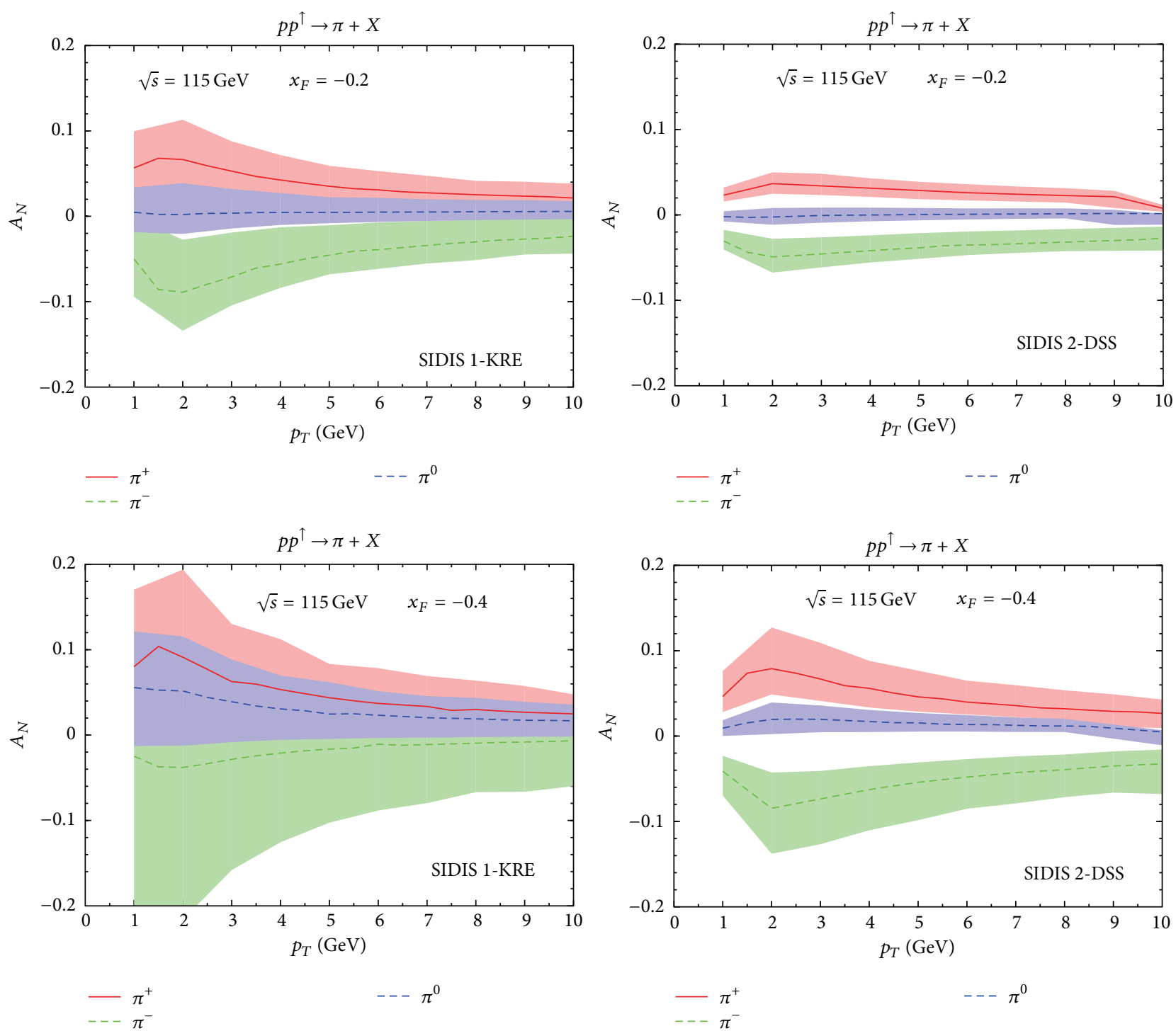

FIgURE 1: Our theoretical estimates for $A_{N}$ versus $p_{T}$ at $\sqrt{s}=115 \mathrm{GeV}, x_{F}=-0.2$ (upper plots), and $x_{F}=-0.4$ (lower plots) for inclusive $\pi^{ \pm}$ and $\pi^{0}$ production in $p p^{\uparrow} \rightarrow \pi X$ processes, computed according to (6)-(8) of the text. The contributions from the Sivers and the Collins effects are added together. The computation is performed adopting the Sivers and Collins functions of [20, 22] (SIDIS 1-KRE, left panels) and of $[21,23]$ (SIDIS 2-DSS, right panels). The overall statistical uncertainty band, also shown, is the envelope of the two independent statistical uncertainty bands obtained following the procedure described in Appendix A of [21].

correction to one of the product of amplitudes). The QCD scale is chosen as $Q=p_{T}$.

The denominator of (1) or (6) is twice the unpolarised cross section and is given in our TMD factorisation by the same expression as in (7), where one simply replaces the factor $\Delta^{N} f_{a / p^{\uparrow}} \cos \left(\phi_{a}\right)$ with $2 f_{a / p}$.

\section{2. $A_{N}$ for Single Pion, Jet, and Direct Photon Production}

We present here our results for $A_{N}$, (1), based on our GPM scheme, (6), (7), and (8). The TMDs which enter in these equations are those extracted from the analysis of SemiInclusive Deep Inelastic (SIDIS) and $e^{+} e^{-}$data [20-23], adopting simple factorised forms, which we recall here.
For the unpolarised TMD partonic distributions and fragmentation functions we have, respectively,

$$
\begin{aligned}
f_{q / p}\left(x, k_{\perp}\right)=f_{q / p}(x) \frac{e^{-k_{\perp}^{2} /\left\langle k_{\perp}^{2}\right\rangle}}{\pi\left\langle k_{\perp}^{2}\right\rangle} & \\
D_{h / q}\left(z, p_{\perp}\right)=D_{h / q}(z) \frac{e^{-p_{\perp}^{2} /\left\langle p_{\perp}^{2}\right\rangle}}{\pi\left\langle p_{\perp}^{2}\right\rangle} & \left\langle k_{\perp}^{2}\right\rangle=0.25 \mathrm{GeV}^{2}, \\
& \left\langle p_{\perp}^{2}\right\rangle=0.20 \mathrm{GeV}^{2} .
\end{aligned}
$$

The Sivers function is parameterised as

$$
\Delta^{N} f_{q / p^{\uparrow}}\left(x, k_{\perp}\right)=2 \mathscr{N}_{q}^{S}(x) f_{q / p}(x) h\left(k_{\perp}\right) \frac{e^{-k_{\perp}^{2} /\left\langle k_{\perp}^{2}\right\rangle}}{\pi\left\langle k_{\perp}^{2}\right\rangle},
$$



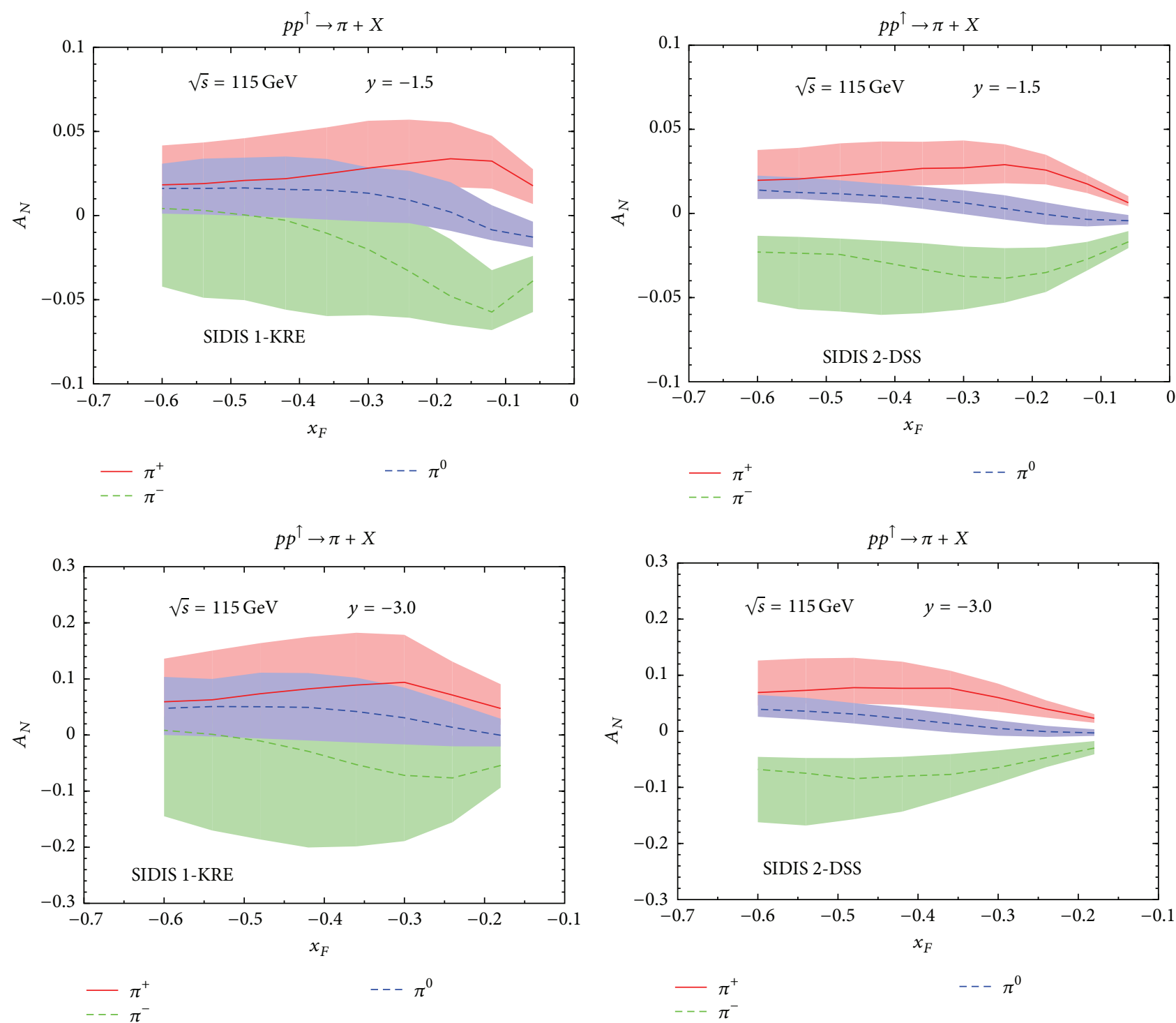

Figure 2: Our theoretical estimates for $A_{N}$ versus $x_{F}$ at $\sqrt{s}=115 \mathrm{GeV}, y=-1.5$ (upper plots), and $y=-3.0$ (lower plots) for inclusive $\pi^{ \pm}$ and $\pi^{0}$ production in $p p^{\uparrow} \rightarrow \pi X$ processes, computed according to (6)-(8) of the text. The contributions from the Sivers and the Collins effects are added together. The computation is performed adopting the Sivers and Collins functions of [20, 22] (SIDIS 1-KRE, left panels) and of [21,23] (SIDIS 2-DSS, right panels). The overall statistical uncertainty band, also shown, is the envelope of the two independent statistical uncertainty bands obtained following the procedure described in Appendix A of [21].

where

$$
\mathcal{N}_{q}^{S}(x)=N_{q}^{S} x^{\alpha_{q}}(1-x)^{\beta_{q}} \frac{\left(\alpha_{q}+\beta_{q}\right)^{\left(\alpha_{q}+\beta_{q}\right)}}{\alpha_{q}^{\alpha_{q}} \beta_{q}^{\beta_{q}}},
$$

with $\left|N_{q}^{S}\right| \leq 1$, and

$$
h\left(k_{\perp}\right)=\sqrt{2 e} \frac{k_{\perp}}{M} e^{-k_{\perp}^{2} / M^{2}}
$$

Similarly, the quark transversity distribution, $\Delta_{T} q\left(x, k_{\perp}\right)$, and the Collins fragmentation function, $\Delta^{N} D_{h / q^{\uparrow}}\left(z, p_{\perp}\right)$, have been parametrized as follows:

$$
\Delta_{T} q\left(x, k_{\perp}\right)=\frac{1}{2} \mathcal{N}_{q}^{T}(x)\left[f_{q / p}(x)+\Delta q(x)\right] \frac{e^{-k_{\perp}^{2} /\left\langle k_{\perp}^{2}\right\rangle}}{\pi\left\langle k_{\perp}^{2}\right\rangle},
$$

$$
\Delta^{N} D_{h / q^{\uparrow}}\left(z, p_{\perp}\right)=2 \mathscr{N}_{q}^{C}(z) D_{h / q}(z) h\left(p_{\perp}\right) \frac{e^{-p_{\perp}^{2} /\left\langle p_{\perp}^{2}\right\rangle}}{\pi\left\langle p_{\perp}^{2}\right\rangle}
$$

where $\Delta q(x)$ is the usual collinear quark helicity distribution,

$$
\begin{aligned}
& \mathcal{N}_{q}^{T}(x)=N_{q}^{T} x^{a_{q}}(1-x)^{b_{q}} \frac{\left(a_{q}+b_{q}\right)^{\left(a_{q}+b_{q}\right)}}{a_{q}^{a_{q}} a_{q}^{b_{q}}}, \\
& \mathcal{N}_{q}^{C}(z)=N_{q}^{C} z^{\gamma_{q}}(1-z)^{\delta_{q}} \frac{\left(\gamma_{q}+\delta_{q}\right)^{\left(\gamma_{q}+\delta_{q}\right)}}{\gamma_{q}^{\gamma_{q}} \delta_{q}^{\delta_{q}}},
\end{aligned}
$$



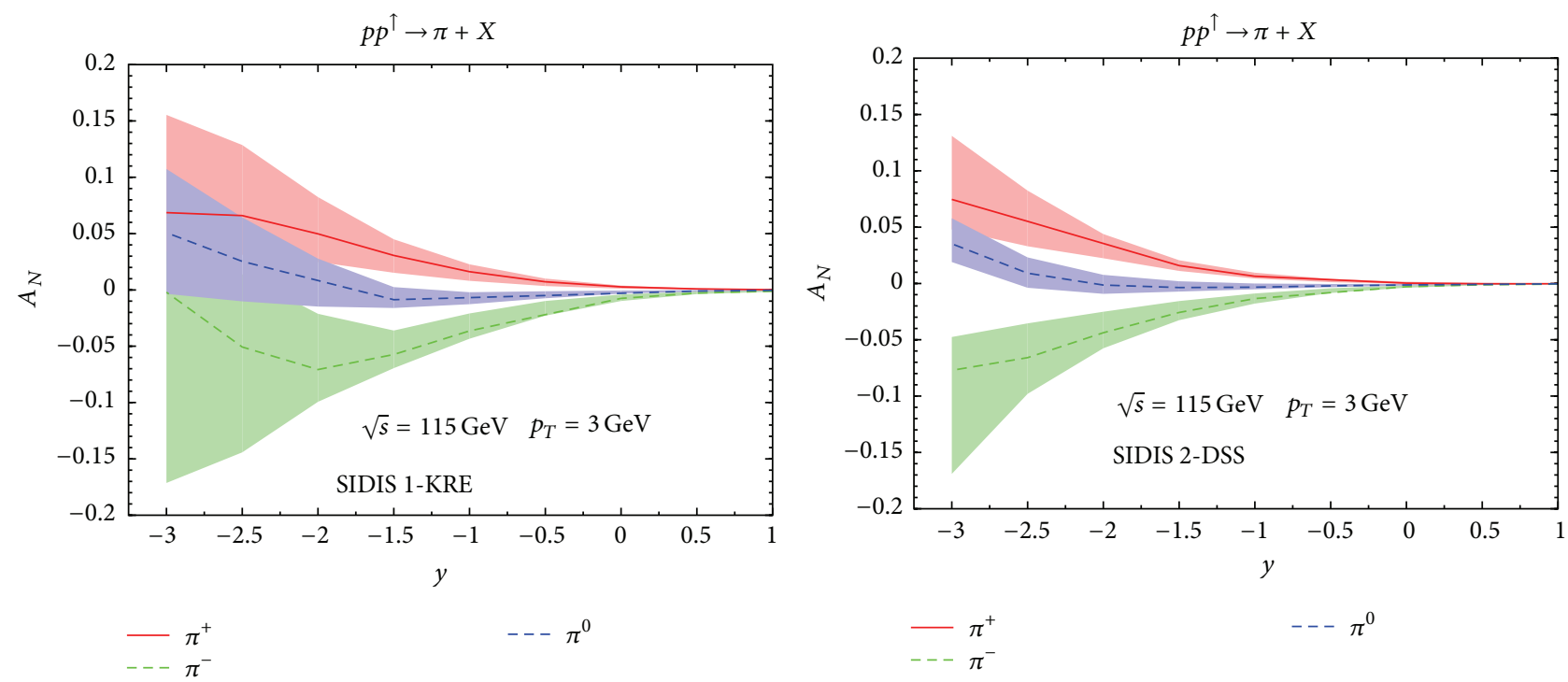

Figure 3: Our theoretical estimates for $A_{N}$ versus $y$ at $\sqrt{s}=115 \mathrm{GeV}$ and $p_{T}=3 \mathrm{GeV}$, for inclusive $\pi^{ \pm}$and $\pi^{0}$ production in $p p^{\uparrow} \rightarrow$ $\pi X$ processes, computed according to (6)-(8) of the text. The contributions from the Sivers and the Collins effects are added together. The computation is performed adopting the Sivers and Collins functions of [20, 22] (SIDIS 1-KRE, left panel) and of [21, 23] (SIDIS 2-DSS, right panel). The overall statistical uncertainty band, also shown, is the envelope of the two independent statistical uncertainty bands obtained following the procedure described in Appendix A of [21].

with $\left|N_{q}^{T(C)}\right| \leq 1$, and

$$
h\left(p_{\perp}\right)=\sqrt{2 e} \frac{p_{\perp}}{M_{c}} e^{-p_{\perp}^{2} / M_{c}^{2}}
$$

All details concerning the motivations for such a choice, the values of the parameters, and their derivation can be found in [20-23]. We do not repeat them here, but in the caption of each figure we will give the corresponding references which allow fixing all necessary values.

We present our results on $A_{N}$ for the process $p p^{\uparrow} \rightarrow$ $\pi X$ at the expected AFTER@LHC energy $(\sqrt{s}=115 \mathrm{GeV})$ in Figures 1-3. Following $[1,2]$, our results are given for two possible choices of the SIDIS TMDs and are shown as function of $p_{T}$ at two fixed $x_{F}$ values (Figure 1), as function of $x_{F}$ at two fixed rapidity $y$ values (Figure 2 ) and as function of rapidity at one fixed $p_{T}$ value (Figure 3 ). $x_{F}$ is the usual Feynman variable defined as $x_{F}=2 p_{L} / \sqrt{s}$ where $p_{L}=\left(\mathbf{p}_{h}\right)_{z}$ is the $z$-component of the final hadron momentum. Notice that, in our chosen reference frame, a forward production, with respect to the polarised proton, means negative values of $x_{F}$. The uncertainty bands reflect the uncertainty in the determinations of the TMDs and are computed according to the procedure explained in the appendix of [21]. More information can be found in the figure captions.

Notice that, for both our choices of the Sivers functions, the gluon Sivers distributions are taken to be vanishing, as suggested by data $[21,24]$. Gluon channels contribute instead to the unpolarised cross sections, in the denominator of (1) or (6). For the unpolarised partonic distributions we adopt the GRV98LO PDF set [25] and for the fragmentation functions the DSS set from [26] and the Kretzer (KRE) set from [27].
The analogous results for the single direct photon are shown in Figures $4-6$ (where $x_{F}=2\left(\mathbf{p}_{\text {jet }}\right)_{z} / \sqrt{s}$ ) and those for the single jet production in Figures 7-9 $\left(x_{F}=2\left(\mathbf{p}_{\gamma}\right)_{z} / \sqrt{s}\right)$. In these cases, obviously, there is no fragmentation process and only the Sivers effect contributes to $A_{N}$, with $D_{h / c}\left(z, p_{\perp}\right)$ simply replaced by $\delta(z-1) \delta^{2}\left(\mathbf{p}_{\perp}\right)$ in (7) (see [2] for further details). In our leading order treatment the jet coincides with a single final parton. Notice that for a jet production we have all the same QCD subprocesses which contribute to hadron production, while for a direct photon production the basic partonic subprocesses are the Compton scattering $g q(\bar{q}) \rightarrow$ $\gamma q(\bar{q})$ and the annihilation process $q \bar{q} \rightarrow \gamma g[28]$.

\section{3. $A_{N}$ for Drell-Yan Processes}

Drell-Yan (D-Y) processes are expected to play a crucial role in our understanding of the origin, at the partonic level, of TSSAs. For such processes, like for SIDIS processes and contrary to single hadron production, the TMD factorisation has been proven to hold, so that there is a general consensus that the Sivers effect should be visible via TSSAs in D-Y [2932]. The widely accepted interpretation of the QCD origin of TSSAs as final or initial state interactions of the scattering partons [33] leads to the conclusion that the Sivers function has opposite signs in SIDIS and D-Y processes [34], which remains to be seen.

Predictions for Sivers $A_{N}$ in D-Y and at different possible experiments were given in [35], which we follow here.

In [35] predictions were given for the $p^{\uparrow} p \rightarrow \ell^{+} \ell^{-} X \mathrm{D}-\mathrm{Y}$ process in the $p^{\uparrow}-p$ c.m. frame, in which one observes the four-momentum $q$ of the final $\ell^{+} \ell^{-}$pair. Notice that $q^{2}=M^{2}$ is the large scale in the process, while $q_{T}=\left|\mathbf{q}_{T}\right|$ is 

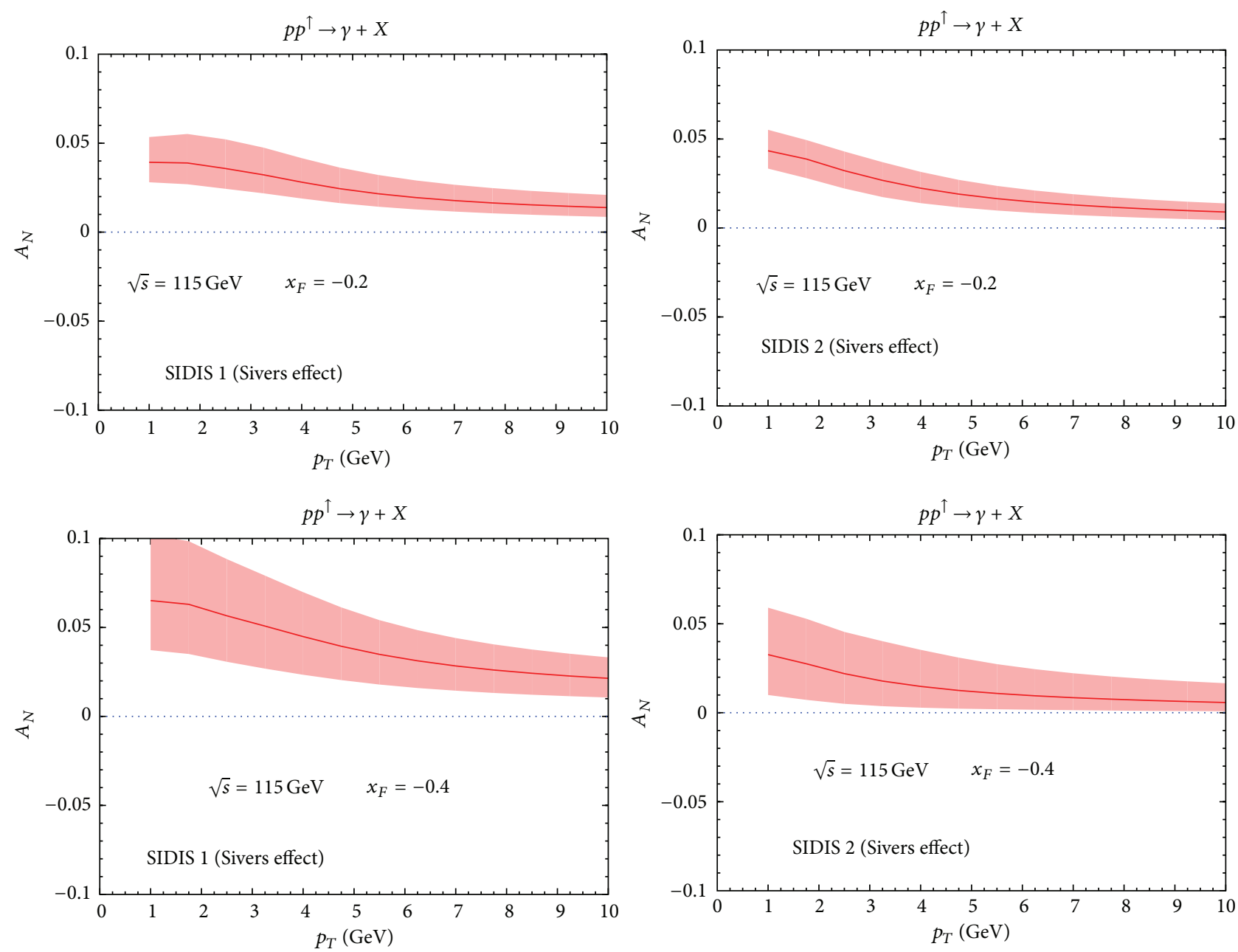

FIgURE 4: Our theoretical estimates for $A_{N}$ versus $p_{T}$ at $\sqrt{s}=115 \mathrm{GeV}, x_{F}=-0.2$ (upper plots), and $x_{F}=-0.4$ (lower plots) for inclusive photon production in $p p^{\uparrow} \rightarrow \gamma X$ processes, computed according to (6) and (7) of the text. Only the Sivers effect contributes. The computation is performed adopting the Sivers functions of [20] (SIDIS 1, left panels) and of [21] (SIDIS 2, right panels). The overall statistical uncertainty band, also shown, is obtained following the procedure described in Appendix A of [21].

the small one. In order to collect data at all azimuthal angles, one defines the weighted spin asymmetry:

$$
\begin{aligned}
A_{N}^{\sin \left(\phi_{\gamma}-\phi_{S}\right)} \equiv & \frac{\int_{0}^{2 \pi} d \phi_{\gamma}\left[d \sigma^{\uparrow}-d \sigma^{\downarrow}\right] \sin \left(\phi_{\gamma}-\phi_{S}\right)}{(1 / 2) \int_{0}^{2 \pi} d \phi_{\gamma}\left[d \sigma^{\uparrow}+d \sigma^{\downarrow}\right]}, \\
& =\frac{\int d \phi_{\gamma}\left[\sum_{q} e_{q}^{2} \int d^{2} \mathbf{k}_{\perp 1} d^{2} \mathbf{k}_{\perp 2} \delta^{2}\left(\mathbf{k}_{\perp 1}+\mathbf{k}_{\perp 2}-\mathbf{q}_{T}\right) \Delta^{N} f_{q / p^{\top}}\left(x_{1}, \mathbf{k}_{\perp 1}\right) f_{\bar{q} / p}\left(x_{2}, k_{\perp 2}\right)\right] \sin \left(\phi_{\gamma}-\phi_{S}\right)}{\int d \phi_{\gamma}\left[\sum_{q} e_{q}^{2} \int d^{2} \mathbf{k}_{\perp 1} d^{2} \mathbf{k}_{\perp 2} \delta^{2}\left(\mathbf{k}_{\perp 1}+\mathbf{k}_{\perp 2}-\mathbf{q}_{T}\right) f_{q / p}\left(x_{1}, k_{\perp 1}\right) f_{\bar{q} / p}\left(x_{2}, k_{\perp 2}\right)\right]},
\end{aligned}
$$

where $\phi_{\gamma}$ and $\phi_{S}$ are, respectively, the azimuthal angle of the $\ell^{+} \ell^{-}$pair and of the proton transverse spin and we have defined (see (2))

$$
\begin{aligned}
\Delta^{N} f_{q / p^{\uparrow}}\left(x, \mathbf{k}_{\perp}\right) & \equiv \Delta^{N} f_{q / p^{\uparrow}}\left(x, k_{\perp}\right) \mathbf{S} \cdot\left(\widehat{\mathbf{P}} \times \widehat{\mathbf{k}}_{\perp}\right) \\
& =\widehat{f}_{q / p^{\uparrow}}\left(x, \mathbf{k}_{\perp}\right)-\widehat{f}_{q / p^{\perp}}\left(x, \mathbf{k}_{\perp}\right) .
\end{aligned}
$$

Adopting for the unpolarised TMD and the Sivers function the same expressions as in (10) and (12)-(14) allows, at $\mathcal{O}\left(k_{\perp} / M\right)$, an analytical integration of the numerator and denominator of (19), resulting in a simple expression for the asymmetry $A_{N}^{\sin \left(\phi_{y}-\phi_{S}\right)}[35]$. 

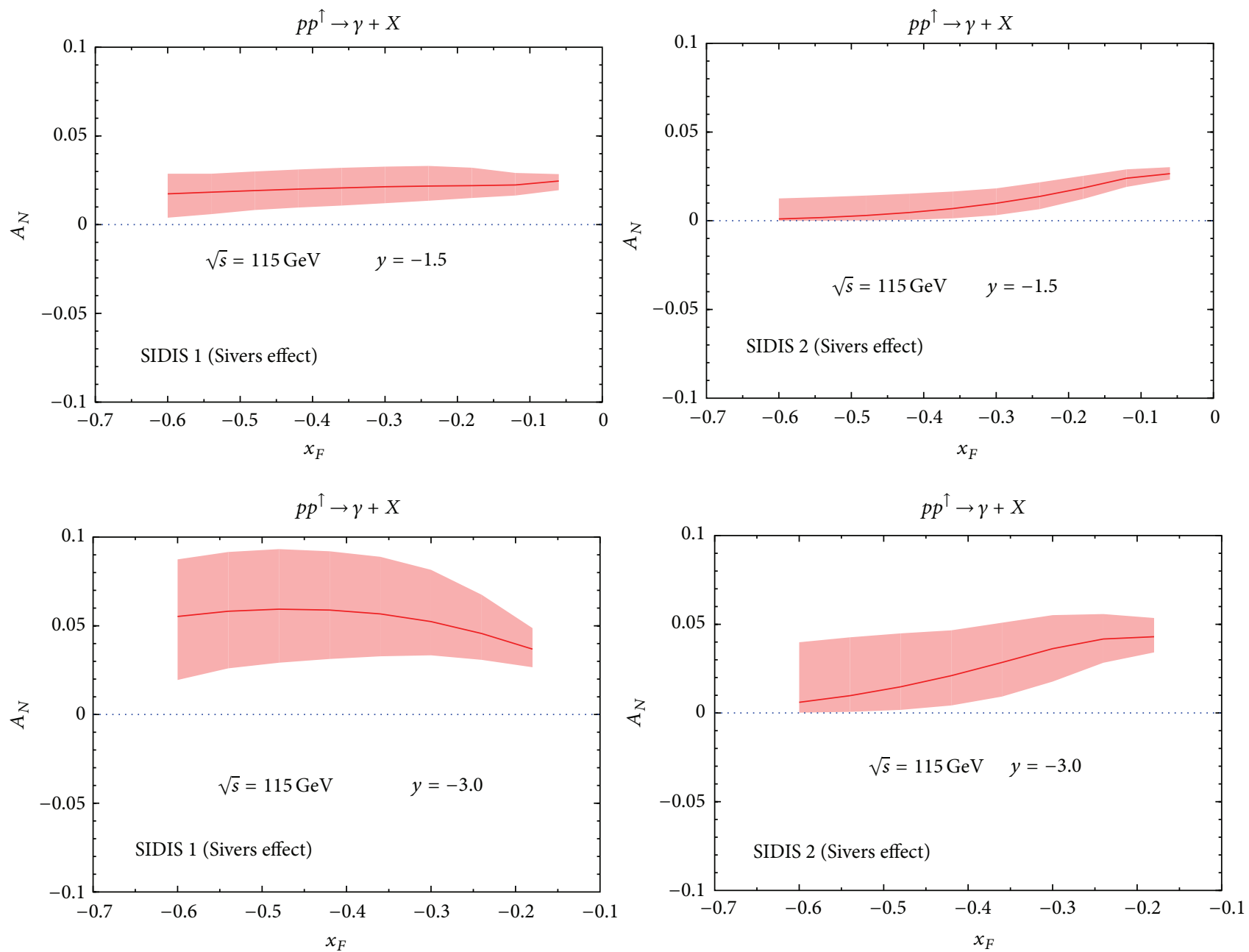

FIGURE 5: Our theoretical estimates for $A_{N}$ versus $x_{F}$ at $\sqrt{s}=115 \mathrm{GeV}, y=-1.5$ (upper plots), and $y=-3.0$ (lower plots) for inclusive photon production in $p p^{\uparrow} \rightarrow \gamma X$ processes, computed according to (6) and (7) of the text. Only the Sivers effect contributes. The computation is performed adopting the Sivers functions of [20] (SIDIS 1, left panels) and of [21] (SIDIS 2, right panels). The overall statistical uncertainty band, also shown, is obtained following the procedure described in Appendix A of [21].
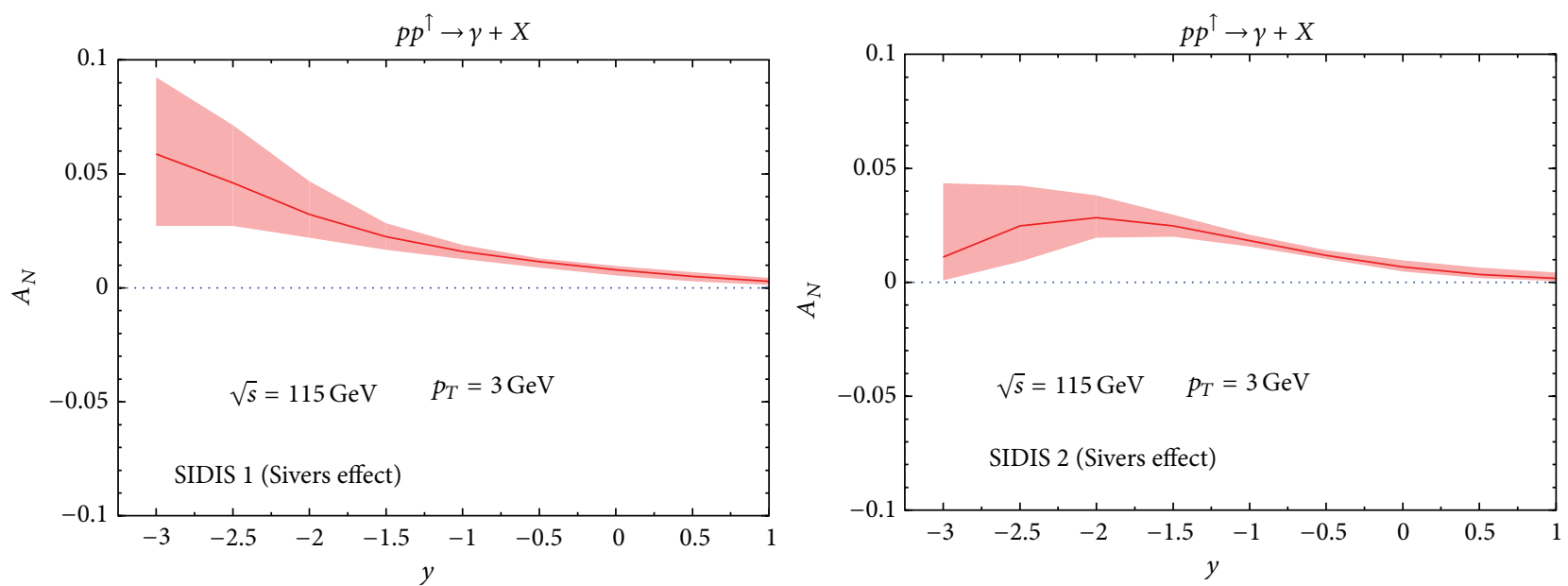

Figure 6: Our theoretical estimates for $A_{N}$ versus $y$ at $\sqrt{s}=115 \mathrm{GeV}$ and $p_{T}=3 \mathrm{GeV}$, for inclusive photon production in $p p^{\uparrow} \rightarrow \gamma X$ processes, computed according to (6)-(8) of the text. Only the Sivers effects contributes. The computation is performed adopting the Sivers functions of [20] (SIDIS 1, left panel) and of [21] (SIDIS 2, right panel). The overall statistical uncertainty band, also shown, is the envelope of the two independent statistical uncertainty bands obtained following the procedure described in Appendix A of [21]. 

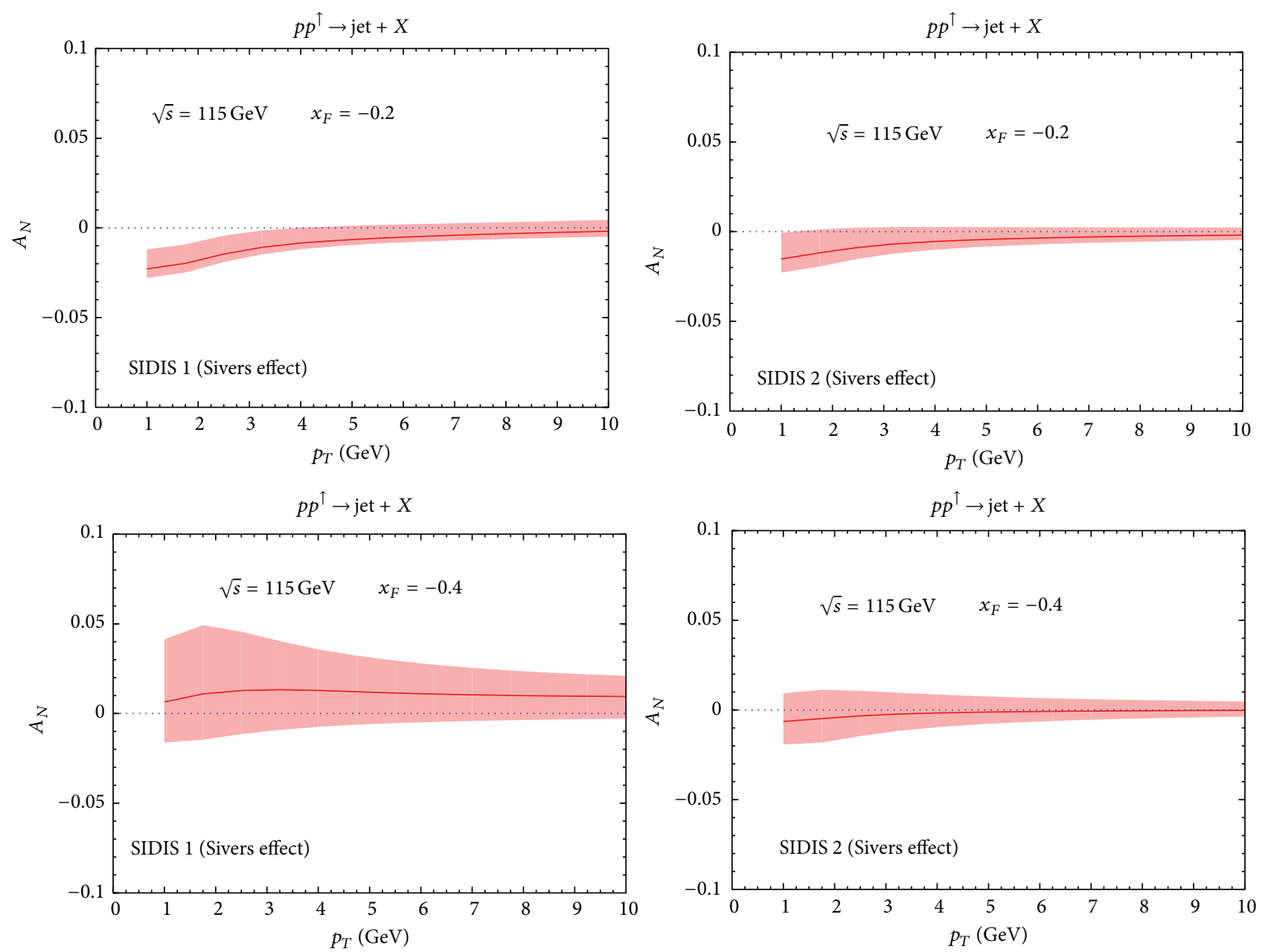

Figure 7: Our theoretical estimates for $A_{N}$ versus $x_{F}$ at $\sqrt{s}=115 \mathrm{GeV}, x_{F}=-0.2$ (upper plots), and $x_{F}=-0.4$ (lower plots) for inclusive single jet production in $p p^{\uparrow} \rightarrow$ jet $X$ processes, computed according to (6) and (7) of the text. Only the Sivers effect contributes. The computation is performed adopting the Sivers functions of [20] (SIDIS 1, left panels) and of [21] (SIDIS 2, right panels). The overall statistical uncertainty band, also shown, is obtained following the procedure described in Appendix A of [21].

Notice that we consider here the $p p^{\uparrow} \rightarrow \ell^{+} \ell^{-} X \mathrm{D}-\mathrm{Y}$ process in the $p-p^{\uparrow}$ c.m. frame. For such a process the TSSA is given by [35]

$$
\begin{aligned}
& A_{N}^{\sin \left(\phi_{\gamma}-\phi_{S}\right)}\left(p p^{\uparrow} \longrightarrow \gamma^{*} X ; x_{F}, M, q_{T}\right) \\
& \quad=-A_{N}^{\sin \left(\phi_{\gamma}-\phi_{S}\right)}\left(p^{\uparrow} p \longrightarrow \gamma^{*} X ;-x_{F}, M, q_{T}\right) .
\end{aligned}
$$

Our results for the Sivers asymmetry $A_{N}^{\sin \left(\phi_{\gamma}-\phi_{S}\right)}$ at AFTER@LHC, obtained following [35], (21) and using the SIDIS extracted Sivers function reversed in sign, are shown in Figure 10. Further details can be found in the captions of these figures.

\section{Comments and Conclusions}

Some final comments and further details might help in understanding the importance of the measurements of the TSSAs at AFTER@LHC: (i) Most predictions given show clear asymmetries, sufficiently large as to be easily measurable, given the expected performance of AFTER@LHC [11]. The uncertainty bands reflect the uncertainty in the extraction of the Sivers and transversity functions from SIDIS data, which are focused on small and intermediate $x$ values $(x \leq 0.3)$; in fact the bands grow larger at larger values of $\left|x_{F}\right|$.

(ii) The values of $A_{N}$ found for pion production can be as large as $10 \%$ for $\pi^{ \pm}$, while they are smaller for $\pi^{0}$. They result from the sum of the Sivers and the Collins effects. The relative importance of the two contributions varies according to the kinematical regions and the set of distributions and fragmentation functions adopted. As a tendency, the contribution from the Sivers effect is larger than the Collins contribution with the SIDIS 1-KRE set, while the opposite is true for the SIDIS 2-DSS set. 

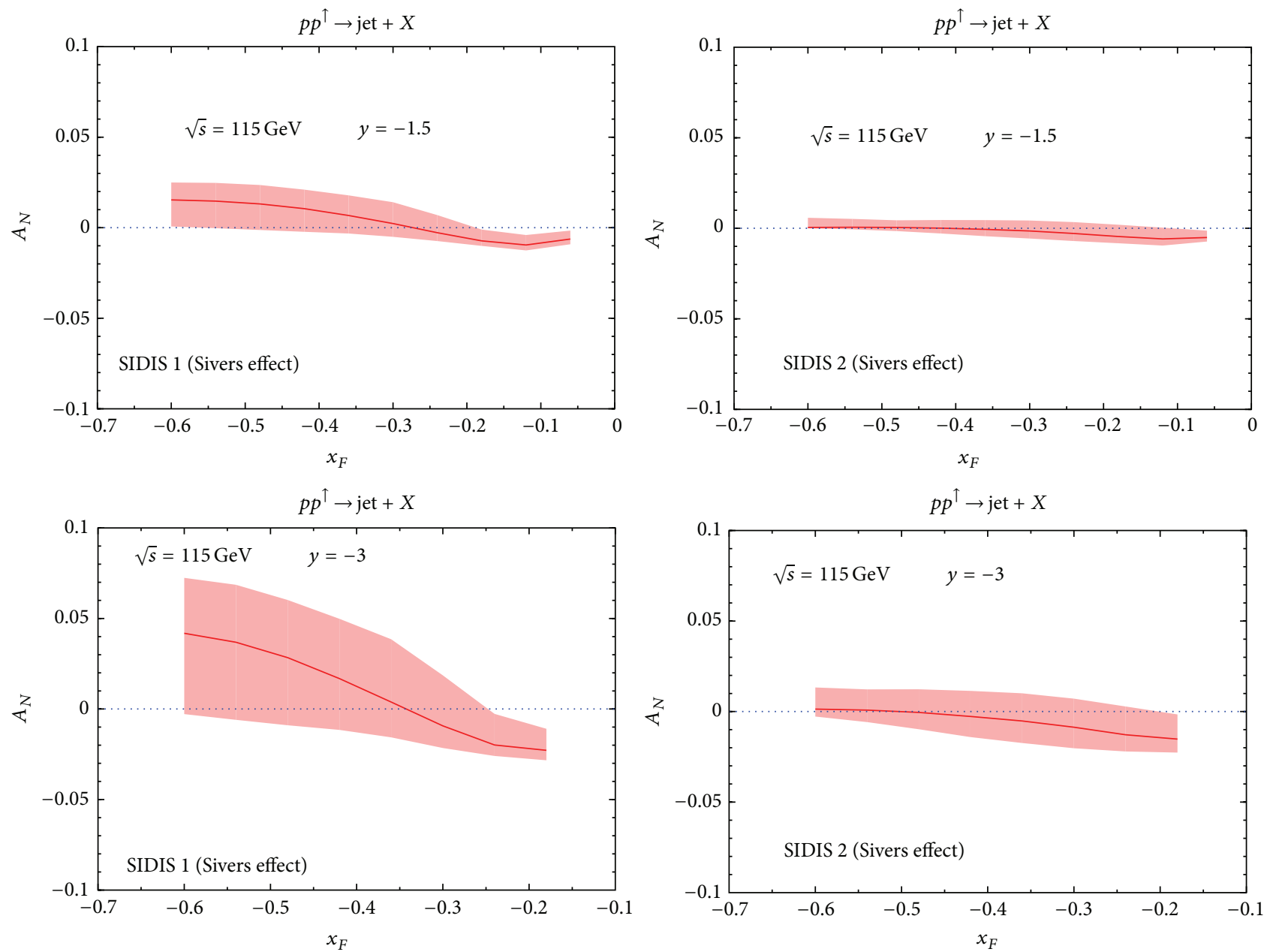

Figure 8: Our theoretical estimates for $A_{N}$ versus $x_{F}$ at $\sqrt{s}=115 \mathrm{GeV}, y=-1.5$ (upper plots), and $y=-3.0$ (lower plots) for inclusive single jet production in $p p^{\uparrow} \rightarrow$ jet $X$ processes, computed according to (6) and (7) of the text. Only the Sivers effect contributes. The computation is performed adopting the Sivers functions of [20] (SIDIS 1, left panels) and of [21] (SIDIS 2, right panels). The overall statistical uncertainty band, also shown, is obtained following the procedure described in Appendix A of [21].
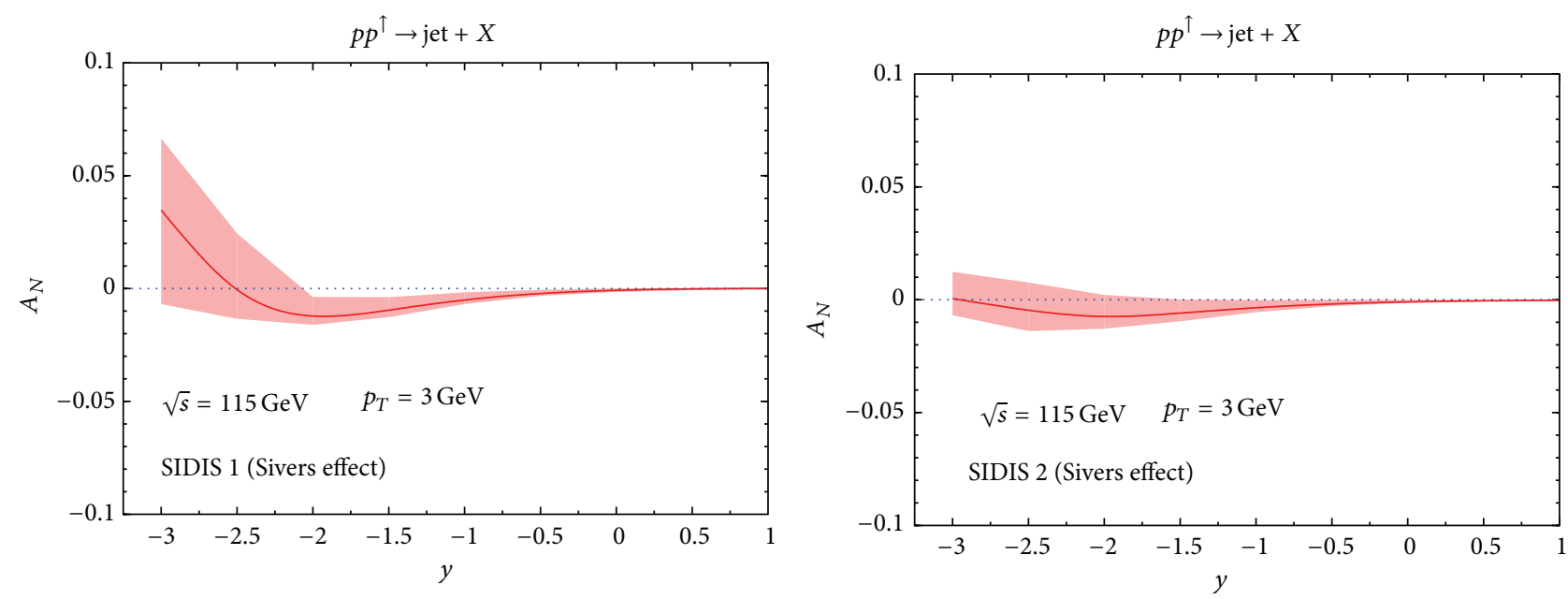

FIGURE 9: Our theoretical estimates for $A_{N}$ versus $y$ at $\sqrt{s}=115 \mathrm{GeV}$ and $p_{T}=3 \mathrm{GeV}$, for inclusive single jet production in $p p^{\uparrow} \rightarrow$ jet $X$ processes, computed according to (6)-(8) of the text. Only the Sivers effect contributes. The computation is performed adopting the Sivers functions of [20] (SIDIS 1, left panel) and of [21] (SIDIS 2, right panel). The overall statistical uncertainty band, also shown, is the envelope of the two independent statistical uncertainty bands obtained following the procedure described in Appendix A of [21]. 

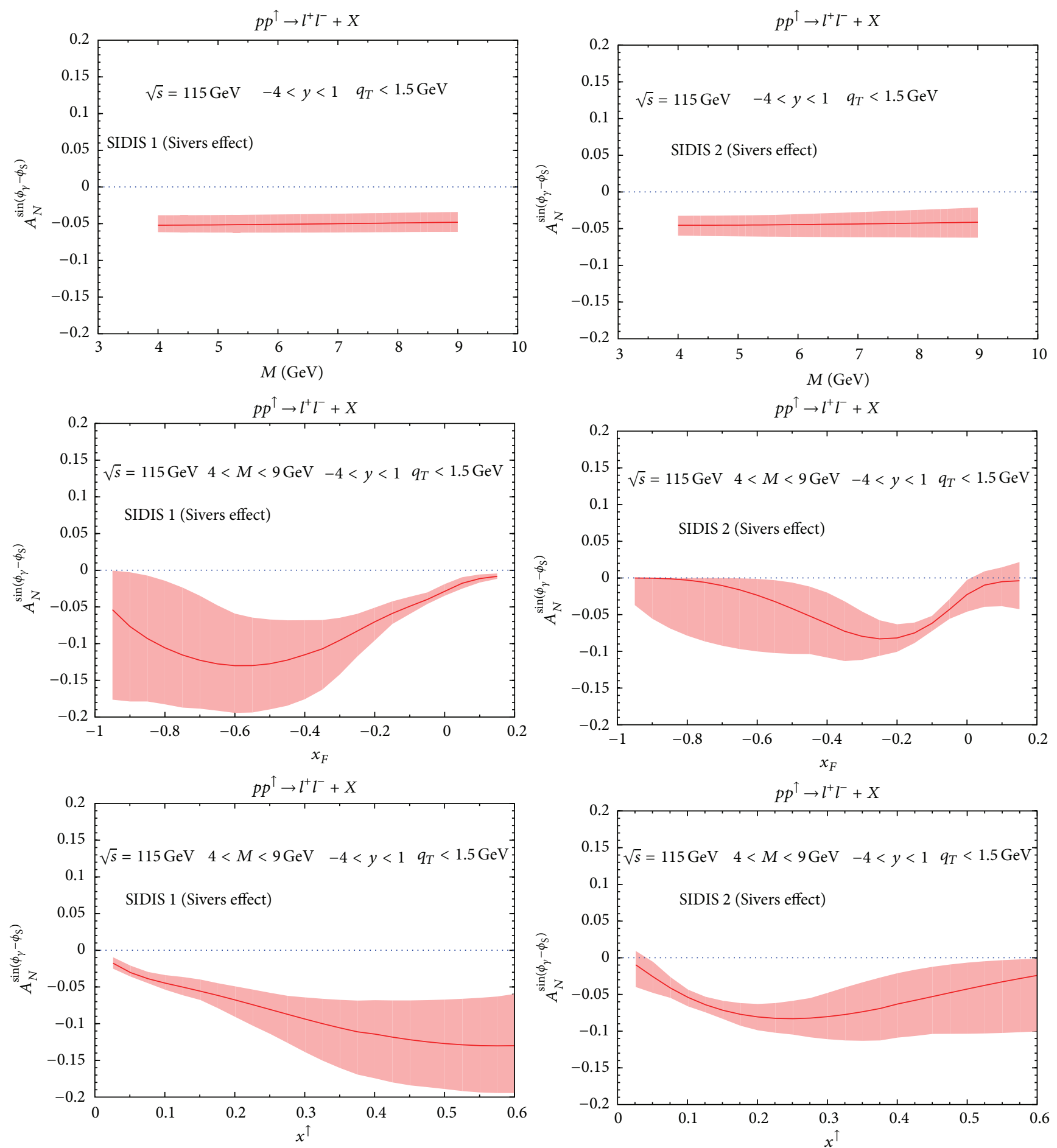

FIGURE 10: Our theoretical estimates for $A_{N}^{\left(\phi_{\gamma}-\phi_{S}\right)}$ in D-Y processes as expected at AFTER@LHC. Our results are presented as function of $M$ (upper plots), $x_{F}$ (middle plots), and $x$ of the quark inside the polarised proton, $x^{\uparrow}$ (lower plots). The other kinematical variables are either fixed or integrated, as indicated in each figure. They are computed according to [35] and (21), adopting the Sivers functions of [20] (SIDIS 1, left panels) and of [21] (SIDIS 2, right panels), reversed in sign. The overall statistical uncertainty band, also shown, is obtained following the procedure described in Appendix A of [21]. 
The values found here are in agreement, both in sign and qualitative magnitude, with the values found in [10] within the collinear twist-3 (CT-3) approach.

(iii) The results for single photon production are interesting; they isolate the Sivers effect and our predictions show that they can reach values of about $5 \%$, with a reduced uncertainty band. We find positive values of $A_{N}$ as the relative weight of the quark charges leads to a dominance of the $u$ quark and the Sivers functions $\Delta^{N} f_{u / p^{\uparrow}}$ which is positive $[20,21]$.

Our results, obtained within the GPM, have a similar magnitude to those obtained in $[3,10]$, within the CT-3 approach, but have an opposite sign. Thus, a measurement of $A_{N}$ for a single photon production, despite being difficult, would clearly discriminate between the two approaches.

(iv) The values of $A_{N}$ for single jet production, which might be interesting as they also have no contribution from the Collins effect, turn out to be very small and compatible with zero, due to a strong cancellation between the $u$ and $d$ quark contributions. The same result is found in [10].

(v) A measurement of $A_{N}^{\sin \left(\phi_{\gamma}-\phi_{S}\right)}$ in D-Y processes at AFTER@LHC is the most interesting one. In such a case the TMD factorisation has been shown to be valid and the Sivers asymmetry should show the expected sign change with respect to SIDIS processes $[33,34]$. Our computations, Figure 10, predict a clear asymmetry, which can be as sizeable as $10 \%$, with a definite sign, even within the uncertainty band.

Both the results of [10] and the results of this paper obtain solid non negligible values for the TSSA $A_{N}$ measurable at the AFTER@LHC experiment. The two sets of results are based on different approaches, respectively, the CT- 3 and the GPM factorisation schemes. While the magnitude of $A_{N}$ is very similar in the two cases, the signs can be different, in particular, the TSSA for a direct photon production, $p p^{\uparrow} \rightarrow$ $\gamma X$, has opposite signs in the two schemes.

In this paper we have also considered azimuthal asymmetries in polarised D-Y processes, related to the Sivers effect. As explained above, in this case, due to the presence of a large and a small scale, like in SIDIS, the TMD factorisation is valid, with the expectation of an opposite sign of the Sivers function in SIDIS and D-Y processes. Also this prediction can be checked at AFTER@LHC.

\section{Conflict of Interests}

The authors declare that there is no conflict of interests regarding the publication of this paper.

\section{Acknowledgments}

M. Anselmino and S. Melis acknowledge support from the "Progetto di Ricerca di Ateneo/CSP" (code TO-Call3-20120103). U. D'Alesio is grateful to the Department of Theoretical
Physics II of the Universidad Complutense of Madrid for the kind hospitality extended to him during the completion of this work.

\section{References}

[1] M. Anselmino, M. Boglione, U. D'Alesio et al., "Role of Collins effect in the single spin asymmetry $A_{N}$ in $p^{\uparrow} p \rightarrow h X$ processes," Physical Review D, vol. 86, Article ID 074032, 2012.

[2] M. Anselmino, M. Boglione, U. D’Alesio, S. Melis, F. Murgia, and A. Prokudin, "Sivers effect and the single spin asymmetry $A_{N}$ in $p^{\uparrow} p \rightarrow h X$ processes," Physical Review D, vol. 88, no. 5, Article ID 054023, 12 pages, 2013.

[3] K. Kanazawa, Y. Koike, A. Metz, and D. Pitonyak, “Transverse single-spin asymmetries in $p^{\uparrow} p \rightarrow \gamma X$ from quark-gluonquark correlations in the proton," Physical Review D, vol. 91, Article ID 014013, 2015.

[4] K. Kanazawa, Y. Koike, A. Metz, and D. Pitonyak, “Towards an explanation of transverse single-spin asymmetries in protonproton collisions: the role of fragmentation in collinear factorization," Physical Review D, vol. 89, no. 11, Article ID 111501, 5 pages, 2014.

[5] J.-W. Qiu and G. F. Sterman, "Single transverse spin asymmetries in direct photon production," Nuclear Physics B, vol. 378, no. 1-2, pp. 52-78, 1992.

[6] J.-W. Qiu and G. F. Sterman, "Single transverse-spin asymmetries in hadronic pion production," Physical Review D, vol. 59, Article ID 014004, 1999.

[7] C. Kouvaris, J.-W. Qiu, W. Vogelsang, and F. Yuan, "Single transverse-spin asymmetry in high transverse momentum pion production in $p p$ collisions," Physical Review D, vol. 74, no. 11, Article ID 114013, 15 pages, 2006.

[8] X. Ji, J.-W. Qiu, W. Vogelsang, and F. Yuan, "Single transversespin asymmetry in Drell-Yan production at large and moderate transverse momentum," Physical Review D, vol. 73, Article ID 094017, 2006.

[9] U. D’Alesio and F. Murgia, "Azimuthal and single spin asymmetries in hard scattering processes," Progress in Particle and Nuclear Physics, vol. 61, no. 2, pp. 394-454, 2008.

[10] K. Kanazawa, Y. Koike, A. Metz, and D. Pitonyak, "Transverse single-spin asymmetries in proton-proton collisions at the AFTER@LHC experiment," http://arxiv.org/abs/1502.04021.

[11] S. J. Brodsky, F. Fleuret, C. Hadjidakis, and J. P. Lansberg, "Physics opportunities of a fixed-target experiment using LHC beams," Physics Reports, vol. 522, no. 4, pp. 239-255, 2013.

[12] L. Massacrier, M. Anselmino, R. Arnaldi et al., "Studies of transverse-momentum-dependent distributions with a fixed-target experiment using the LHC beams (AFTER@LHC)," http://arxiv .org/abs/1502.00984.

[13] D. W. Sivers, "Single-spin production asymmetries from the hard scattering of pointlike constituents," Physical Review D, vol. 41, pp. 83-90, 1990.

[14] D. W. Sivers, "Hard-scattering scaling laws for single-spin production asymmetries," Physical Review D, vol. 43, no. 1, pp. 261263, 1991.

[15] J. C. Collins, "Fragmentation of transversely polarized quarks probed in transverse momentum distributions," Nuclear Physics $B$, vol. 396, no. 1, pp. 161-182, 1993.

[16] M. Anselmino, M. Boglione, U. D’Alesio, E. Leader, S. Melis, and F. Murgia, "General partonic structure for hadronic spin 
asymmetries," Physical Review D, vol. 73, Article ID 014020, 2006.

[17] P. J. Mulders and R. D. Tangerman, “The complete tree-level result up to order $1 / Q$ for polarized deep-inelastic leptoproduction," Nuclear Physics B, vol. 461, no. 1-2, pp. 197-237, 1996.

[18] A. Bacchetta, U. D’Alesio, M. Diehl, and C. A. Miller, "Singlespin asymmetries: the Trento conventions," Physical Review D, vol. 70, no. 11, Article ID 117504, 2004.

[19] M. Anselmino, M. Boglione, U. D'Alesio, E. Leader, and F. Murgia, "Parton intrinsic motion: suppression of the Collins mechanism for transverse single spin asymmetries in $p^{\uparrow} p \rightarrow$ $\pi X$," Physical Review D, vol. 71, Article ID 014002, 2005.

[20] M. Anselmino, M. Boglione, U. D’Alesio, A. Kotzinian, F. Murgia, and A. Prokudin, "Extracting the Sivers function from polarized semi-inclusive deep inelastic scattering data and making predictions," Physical Review D, vol. 72, no. 9, Article ID 094007, 9 pages, 2005.

[21] M. Anselmino, M. Boglione, U. D’Alesio et al., "Sivers effect for pion and kaon production in semi-inclusive deep inelastic scattering," The European Physical Journal A, vol. 39, no. 1, pp. 89-100, 2009.

[22] M. Anselmino, M. Boglione, U. D’Alesio et al., "Transversity and Collins functions from SIDIS and $\mathrm{e}^{+} \mathrm{e}^{-}$data," Physical Review D, vol. 75, Article ID 054032, 2007.

[23] M. Anselmino, M. Boglione, U. D’Alesio et al., "Update on transversity and Collins functions from SIDIS and $\mathrm{e}^{+} \mathrm{e}^{-}$data," Nuclear Physics B-Proceedings Supplements, vol. 191, pp. 98107, 2009.

[24] M. Anselmino, U. D’Alesio, S. Melis, and F. Murgia, "Constraints on the gluon Sivers distribution via transverse single spin asymmetries at midrapidity in $p^{\uparrow} p \rightarrow \pi^{0} X$ processes at BNL RHIC," Physical Review D, vol. 74, no. 9, Article ID 094011, 7 pages, 2006.

[25] M. Gluck, E. Reya, and A. Vogt, "Dynamical parton distributions revisited," The European Physical Journal C, vol. 5, no. 3, pp. 461-470, 1998.

[26] D. de Florian, R. Sassot, and M. Stratmann, "Global analysis of fragmentation functions for pions and kaons and their uncertainties," Physical Review D, vol. 75, Article ID 114010, 2007.

[27] S. Kretzer, "Fragmentation functions from flavor-inclusive and flavor-tagged $\mathrm{e}^{+} \mathrm{e}^{-}$annihilations," Physical Review D, vol. 62, Article ID 054001, 2000.

[28] U. D’Alesio and F. Murgia, "Parton intrinsic motion in inclusive particle production: unpolarized cross sections, single spin asymmetries, and the Sivers effect," Physical Review D, vol. 70, no. 7, Article ID 074009, 22 pages, 2004.

[29] J. C. Collins, D. E. Soper, and G. F. Sterman, "Transverse momentum distribution in Drell-Yan pair and $\mathrm{W}$ and $\mathrm{Z}$ boson production," Nuclear Physics B, vol. 250, no. 1-4, pp. 199-224, 1985.

[30] X.-D. Ji, J.-P. Ma, and F. Yuan, "QCD factorization for spindependent cross sections in DIS and Drell-Yan processes at low transverse momentum," Physics Letters B, vol. 597, no. 3-4, pp. 299-308, 2004.

[31] J. Collins, Foundations of Perturbative QCD, vol. 32 of Cambridge Monographs on Particle Physics, Nuclear Physics and Cosmology, Cambridge University Press, Cambridge, UK, 2011.

[32] M. G. Echevarria, A. Idilbi, and I. Scimemi, "Factorization theorem for Drell-Yan at low $q_{T}$ and transverse-momentum distributions on-the-light-cone," Journal of High Energy Physics, vol. 2012, article 2, 2012.
[33] S. J. Brodsky, D. S. Hwang, and I. Schmidt, "Final-state interactions and single-spin asymmetries in semi-inclusive deep inelastic scattering," Physics Letters B, vol. 530, no. 1-4, pp. 99$107,2002$.

[34] J. C. Collins, "Leading-twist single-transverse-spin asymmetries: Drell-Yan and deep-inelastic scattering," Physics Letters B, vol. 536, no. 1-2, pp. 43-48, 2002.

[35] M. Anselmino, M. Boglione, U. D’Alesio, S. Melis, F. Murgia, and A. Prokudin, "Sivers effect in Drell-Yan processes," Physical Review D, vol. 79, Article ID 054010, 2009. 

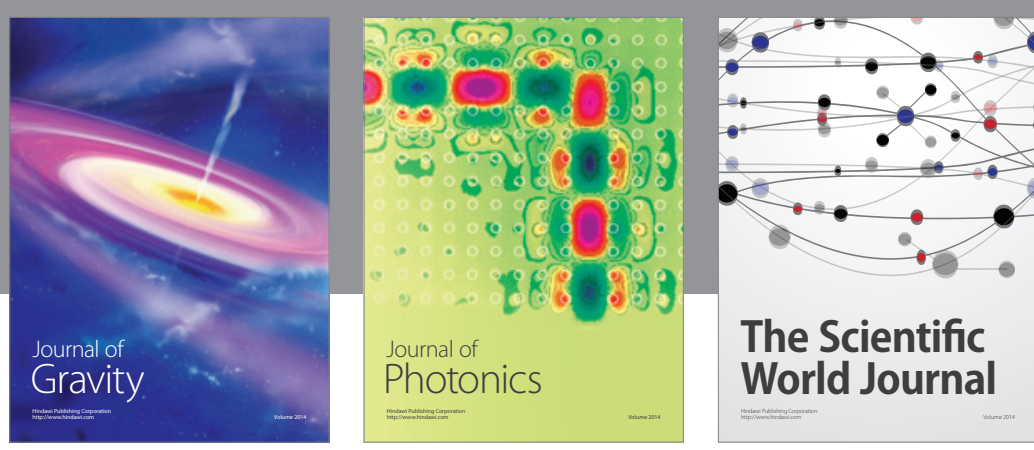

The Scientific World Journal
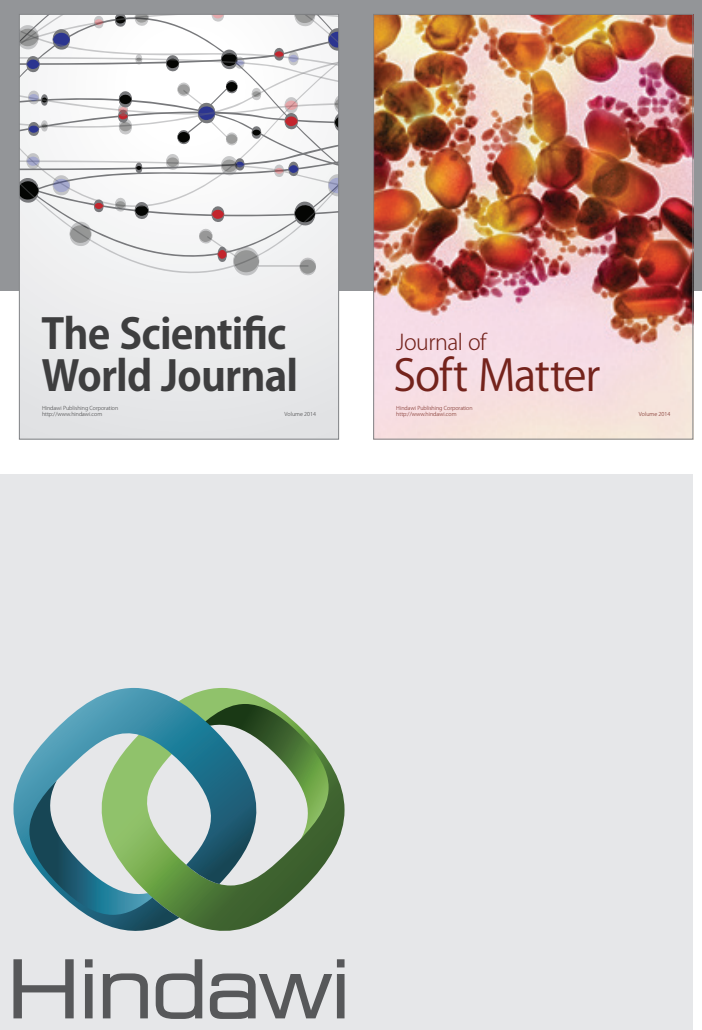

Submit your manuscripts at

http://www.hindawi.com

nternational Journal of

Statistical Mechanics
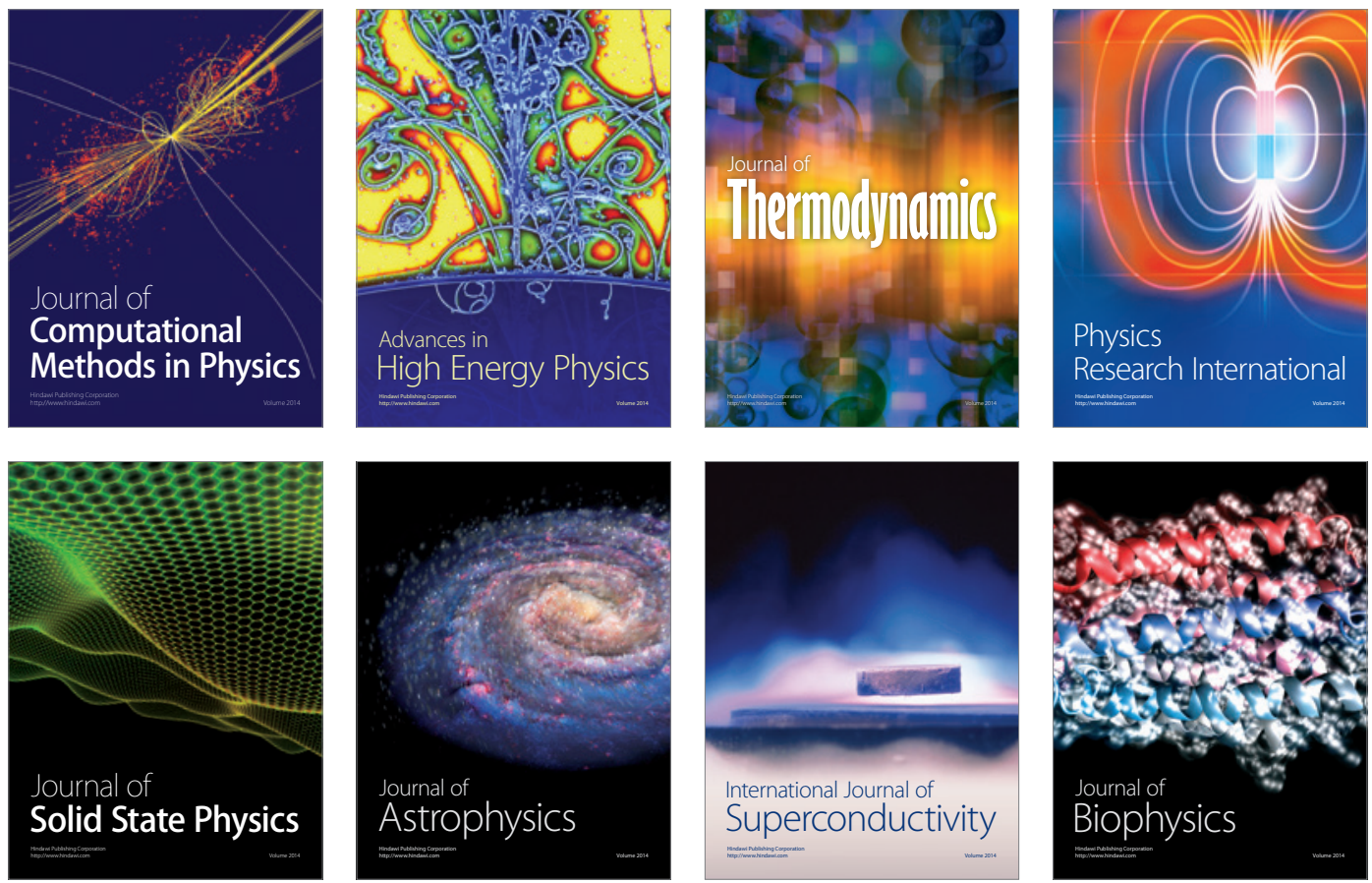
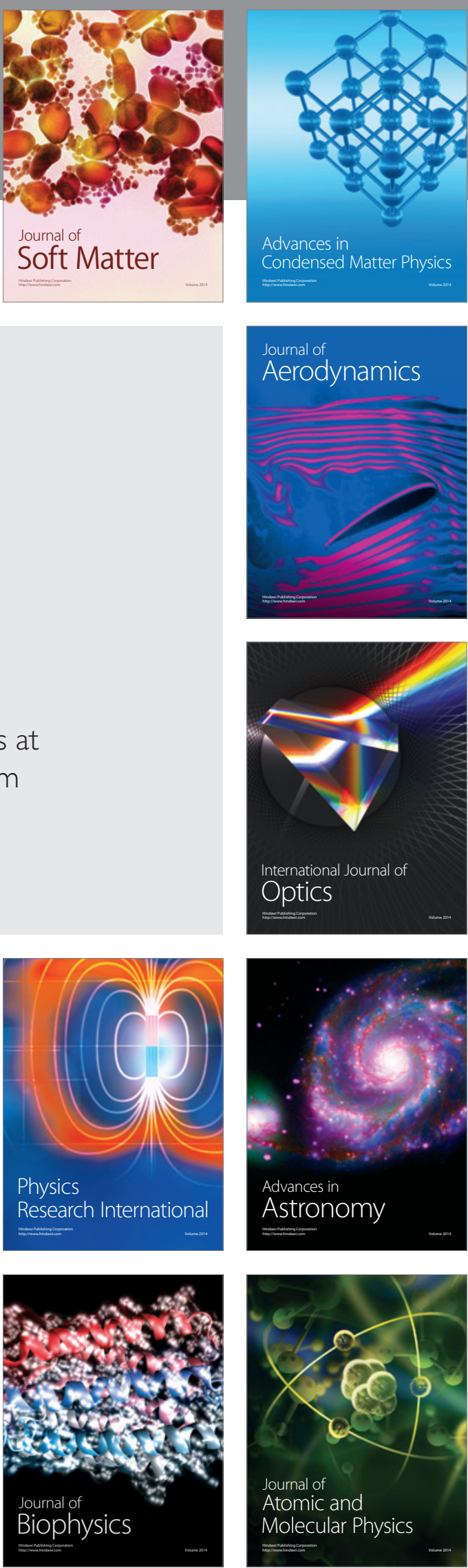